\title{
EXPOSIÇÃO DE CRIANÇAS A AGROTÓXICOS: UMA REVISÃO INTEGRATIVA
}

\author{
Maria Goreti Finkler \\ Universidade Federal da Fronteira Sul \\ Campus Cerro Largo, Brasil \\ maria.finkler@uffs.edu.br \\ lara Denise Endruweit Battisti \\ Profa. Dra. da Universidade Federal da Fronteira Sul \\ Campus Cerro Largo, Brasil \\ iara.battisti@uffs.edu.br \\ Zélia Ferreira Caçador Anastácio \\ Profa. Dra. do CIEC, Instituto de Educação \\ Universidade do Minho, Portugal \\ zeliaf@ie.uminho.pt
}

Fecha de Recepción: 12 Septiembre 2019

Fecha de Admisión: 25 Septiembre 2019

\section{RESUMO}

A agricultura representa uma importante atividade econômica mundial e a busca pelo aumento da produtividade incorporou o uso de agrotóxicos nesta prática. Com isso a exposição a esses produtos químicos e possíveis prejuízos a saúde tornou-se um problema de saúde pública. As crianças são particularmente vulneráveis à exposição a agrotóxicos, devido a fatores de desenvolvimento, dieta e fisiologia. 0 objetivo desse estudo foi realizar uma revisão integrativa em plataforma digital PubMed para levantar e analisar as publicações científicas dos últimos cinco anos sobre a relação da exposição de crianças a agrotóxicos. Após a triagem, 32 estudos foram selecionados de acordo com critério pré-estabelecidos. Os artigos foram resumidos com base nos objetivos, na metodologia e nos resultados. Os delineamentos dos artigos foram: 15 transversais, seis caso-controle, cinco coortes, três coortes prospectivas, dois observacionais e um experimental. As publicações produzidas no período analisado, evidenciam o prejuízo causado sobre a saúde das crianças, tais como aumento de micronúcleos, danos oxidativos ao DNA, leucemia, efeitos neurológicos, asma, rinite alérgica, alterações de tireoide, entre outros agravos a saúde. Portanto, esta revisão integrativa evidenciou que a exposição de crianças a agrotóxicos na zona rural está de fato associado a ocorrência de uma série de agravos a saúde infantil.

Palavras-chave: agroquímicos; saúde infantil; área rural. 


\section{EXPOSIÇÃO DE CRIANÇAS A AGROTÓXICOS: UMA REVISÃO INTEGRATIVA}

\section{ABSTRACT}

Children exposure to agrotoxic: an integrative review. Agriculture represents an important sector in the global economy and to increase productivity, pesticides are utilized. Thus, exposure to these chemicals and possible health damage has become a public health problem. Children are particularly vulnerable to exposure to pesticides due to developmental factors, diet, and physiology. The objective of this study was to perform an integrative review using the PubMed database to survey and analyze the scientific publications of the last five years about children's exposure to pesticides. After the screening, 32 studies were selected according to pre-established criteria. The articles were summarized based on the objectives, methods, and results. The study designs of the articles were: 15 cross- sectional, six case-controls, five cohorts, three prospective cohorts, two observational, and one experimental. In the analyzed period, the studies evidenced the effect on children's health due to pesticides. These health effects included micronucleus increase, oxidative DNA damage, leukemia, neurological effects, asthma, allergic rhinitis, thyroid disorders, among others. Therefore, this integrative review showed that the exposure of children to pesticides in rural areas is in fact associated with the occurrence of several health problems.

Keywords: agrochemicals; children's health; rural área.

\section{INTRODUÇÃO}

As crianças são expostas a agrotóxicos por vias ambientais, em suas casas, escolas, gramados e jardins, assim como pela alimentação e água contaminada, e por vias ocupacionais, durante sua participação nas atividades laborais da família e através do contato com os pais, após terem lidado com estes agentes químicos durante as atividades de trabalho (Sarcinelli, 2003).

0 Brasil é um dos maiores produtores agropecuários do mundo e o segundo país que mais exporta esses produtos. Para manter tal produção, este setor utiliza intensivamente sementes transgênicas e insumos agrícolas, como fertilizantes e agrotóxicos (Pignati et al., 2017). A extensa área de plantio no Brasil proporcionou que o país fosse, em 2010, o maior consumidor mundial per capita desses compostos, representando uma média de mais de cinco quilos de agrotóxicos por brasileiro por ano (Carneiro, Rigotto, Augusto, Friedrich, Búrigo, 2015).

Em 2017 o estado do Rio Grande do Sul figurou entre os três estados brasileiros com maior comercialização de agrotóxicos, com 70.143,6 toneladas de ingrediente ativo, ficando atrás apenas de Mato Grosso e São Paulo (Ibama, 2018). Ainda, conforme relatório divulgado pelo Centro de Vigilância em Saúde da Secretaria Estadual da Saúde (Cevs/Ses) do RS, a região noroeste do estado do RS é a área de maior utilização de agrotóxicos de todo estado e chega a ser empregado 919 $\mathrm{L} \mathrm{km}^{-2}$ ano $^{-1}$ de agrotóxicos (Barreto, Herman e Garibotti, 2012; Cevs/Ses, 2010).

0 destaque no cenário nacional e internacional sobre a intensidade de uso dessas substâncias traz consigo inúmeras preocupações, principalmente de riscos potenciais decorrentes da exposição humana. A saúde de trabalhadores em diversas áreas é ameaçada devido à exposição a esses produtos durante a jornada de trabalho. Além desses, as mulheres em período fértil e as crianças constituem os grupos humanos mais vulneráveis (Carneiro et al., 2015).

As crianças que vivem em ambientes rurais encontram riscos ambientais derivados de atividades de produção agrícola, tanto pela exposição múltipla e contínua quanto pelas condições de trabalho e saúde, que podem agravar consideravelmente os efeitos produzidos por esses contaminantes químicos (Perry, 2003; Karr, 2012; Miller, Marty e Landrigan, 2016).

Em geral, as residências situam-se no meio das lavouras, assim como as escolas se encontram muito próximas a estas áreas, e mesmo as crianças que não se expõem diretamente durante 0 trabalho são alvo da contaminação por várias rotas, como ar, água e solo. Nesse contexto, as crianças 
cujos familiares são produtores e trabalhadores rurais podem sofrer riscos maiores de exposição a agrotóxicos que as da população geral (Sarcinelli, 2003).

A exposição de crianças pode diferir da exposição de adultos, pois a fisiologia e 0 comportamento único da criança podem influenciar na extensão da exposição. As crianças são particularmente sensíveis aos agrotóxicos em decorrência de sua alta permeabilidade intestinal e da imaturidade do seu sistema de detoxificação. Essas diferenças devem ser levadas em consideração quando se avaliam riscos dos agrotóxicos (Carneiro et al., 2015).

Carneiro et al. (2015) relatam um acidente que envolveu a pulverização aérea de agrotóxicos, com repercussões graves, acontecido em 2013, no município de Rio Verde (G0), responsável por contaminar uma escola e provocar efeitos agudos em crianças, professores e servidores. A longo prazo, é possível e, infelizmente, esperado, que esse crime também ocasione efeitos crônicos nas vítimas dessa intoxicação.

Portanto, frente a preocupação com o impacto potencialmente desproporcional que exposições a agrotóxicos podem causar na saúde de crianças e fetos em desenvolvimento, objetivou-se reunir uma compilação de artigos científicos de revistas indexadas na base de dados PubMed referente a exposição de crianças a agrotóxicos, com o intuito de mapear e discutir os materiais e métodos utilizados para averiguar a contaminação de crianças por agrotóxicos e visualizar os efeitos que esta exposição acarreta na saúde das crianças.

\section{OBJETIVOS DA PESQUISA}

0 presente estudo teve como objetivo realizar uma revisão integrativa em plataforma digital e levantar e analisar as publicações científicas dos últimos cinco anos sobre a relação da exposição de crianças a agrotóxicos.

\section{METODOLOGIA E/OU INSTRUMENTOS UTILIZADOS}

Neste trabalho realizou-se um levantamento na base de dados PubMed das produções científicas publicadas nos últimos 5 (2014 - 2019) anos sobre a relação "exposição de crianças a agrotóxicos".

Os estudos foram selecionados através da pesquisa no banco de dados PubMed (https://www.ncbi.nlm.nih.gov/pubmed/advanced) usando as seguintes palavras-chave em inglês e português: ((pesticide OR herbicide OR insecticide OR fungicide OR organophosphate OR carbamate OR carbamates $O R$ agrochemical $O R$ herbicida OR inseticida OR fungicida OR organofosforados OR agrotoxicos OR agroquimico OR carbamato OR carbamatos) AND ("rural people" OR "rural population" OR "rural areas" OR "non-urban" OR rural OR "trabalhador rural" OR agricola OR "populacao rural" OR "areas rurais") AND (criança OR crianças OR bebe OR bebes OR adolescente OR adolescentes OR child OR children OR baby OR babies OR teenager OR teenagers)).

Os artigos originais foram incluídos nesta revisão se foram publicados entre 2014 e 2019 e examinaram a relação de agrotóxicos e crianças da zona rural. Os estudos foram excluídos se (a) não estivessem relacionados aos filhos de trabalhadores rurais ou crianças residentes de áreas rurais; (b) não analisaram agrotóxicos e crianças; (c) foram revisões; (d) analisou a ingestão de agrotóxicos através dos alimentos; (e) eram estudos genéticos; (f) não foram escritos em inglês ou português; (g) não estavam totalmente disponíveis.

\section{RESULTADOS ALCANÇADOS}

Realizou-se uma triagem primária dos títulos, seguida dos resumos a fim de remover os registros que se enquadravam nos critérios de exclusão. Nesta primeira etapa excluiu-se 240 artigos por estes não relacionarem a exposição a agrotóxicos com crianças; envolver estudos com foco no uso 


\section{EXPOSIÇÃO DE CRIANÇAS A AGROTÓXICOS: UMA REVISÃO INTEGRATIVA}

de agrotóxicos em residências para combate de insetos vetores de doenças; não terem sido escritos em inglês ou português; tratarem de revisões; serem estudos genéticos e; não estarem totalmente disponíveis. Posteriormente, foi realizada uma triagem secundária onde se analisou o texto completo. Nesta segunda fase, removeu-se 13 artigos por estes não relacionar exposição a agrotóxicos em crianças que residem em áreas rurais; tratar da exposição a agrotóxicos em mães e por alguns estudos abordarem como foco a exposição de crianças residentes em áreas rurais a metais pesados e outros poluentes. Após a triagem, 32 estudos foram finalmente escolhidos para constituir 0 conjunto. A Figura 1 ilustra a busca na plataforma digital PubMed.

Figura 1. Fluxograma de revisão integrativa.

Fonte: Elaborado pelos autores, 2019.

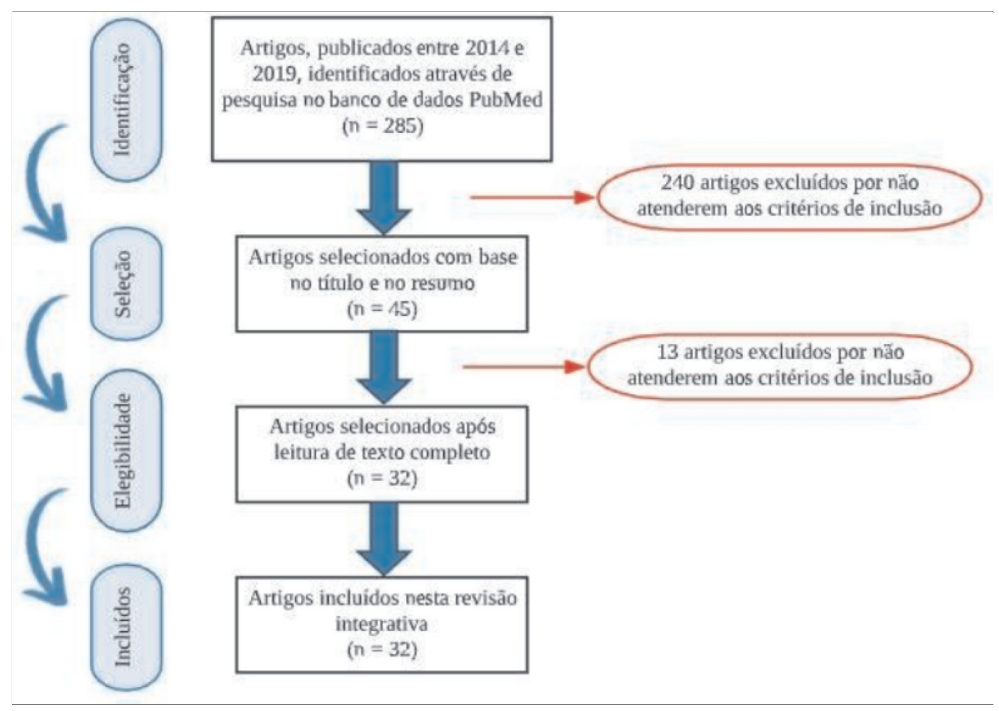

Dos 32 artigos selecionados, apenas cinco deles contemplam estudos realizados no Brasil, cujos artigos apresentavam delineamento de estudo transversal. Os artigos foram provenientes de 22 revistas distintas, sendo que todos foram classificados conforme o sistema brasileiro de avaliação de periódicos Qualis-Periódicos, conforme ilustra a Tabela 1.

Tabela 1

Classificação dos periódicos segundo o sistema brasileiro de avaliação de periódicos Qualis.

\begin{tabular}{|c|c|c|c|c|c|}
\hline \multicolumn{6}{|l|}{ Classificação Qualis } \\
\hline \multirow{2}{*}{ Número de artigos } & A1 & A2 & $\mathrm{B} 1$ & $\mathrm{~B} 2$ & Sem classificação* \\
\hline & 11 & 5 & 9 & 1 & 6 \\
\hline
\end{tabular}

Nota. *Artigos sem classificação na área de saúde coletiva. Fonte: Elaborado pelos autores, 2019.

A maioria dos estudos coletados foram realizados na América do Sul $(10 ; 31,25 \%)$, seguido da América do Norte (8; 25\%), Ásia (6; 18,75\%), Europa (4; 12,5\%) e África $(4 ; 12,5$ 
$\%$ ). Destes estudos, cinco foram realizados nos Estados Unidos da América, outros cinco foram realizados no Brasil, três na Argentina e dois no México, o restante foram realizados em diferentes países. Os delineamentos dos estudos contemplaram 15 transversais, seis caso- controle, cinco coortes, três coortes prospectivas, dois observacionais e um experimental. Como método de estudo, para averiguar a exposição de crianças a agrotóxicos, a maioria das pesquisas fez uso de amostras de urina (13; 40,62 \%) de crianças para analisar a presença ou interferência da exposição a agrotóxicos. Outros estudos utilizaram a coleta de amostras de sangue $(10 ; 31,25$

$\%$ ), cabelo (2; $6,25 \%)$ e mucosa bucal $(1 ; 3,12 \%)$. Ainda, entre esses estudos, muitos aplicaram questionários pertinente ao assunto, respondidos pelos responsáveis das crianças.

\section{DISCUSSÃO}

As publicações produzidas no período de 2014 a 2019 sobre a exposição das crianças a agrotóxicos na área rural, encontradas no presente estudo, evidenciam o prejuízo causado sobre a saúde das crianças, tais como aumento de micronúcleos, malformações congênitas, danos citogenéticos, leucemia, efeitos neurológicos, asma, rinite alérgica, alterações de tireoide, entre outros agravos a saúde.

Um estudo caso-controle realizado por Kapka-Skrzypczak et al. (2019) na Polônia, tinha como objetivo investigar se o dano ao DNA aumentava devido a exposição prolongada a agrotóxicos. A amostra contemplou a participação de 108 crianças (grupo exposto) que viviam em áreas de uso intenso de agrotóxicos e mais 92 crianças (grupo controle) que residiam em uma área agroturística. Através da coleta de amostras de sangue para analisar acetilcolinesterase (AChE) e micronúcleos (MN) obteve-se como resultado que o aumento da exposição de crianças a agrotóxicos aumentou o número de MN nos linfócitos de sangue $(p=0,016)$. Houve aumento do nível de quebra das cadeias de DNA $(p=0,002)$ e dano oxidativo ao DNA $(p<0,001)$. Verificou-se correlação negativa entre o nível de quebras na fita de DNA e a atividade da AChE no grupo exposto. Deste modo, verificou-se efeitos biológicos significativos apenas nas crianças expostas a agrotóxicos.

Um estudo de coorte realizado durante julho e outubro de 2012 em Yakima, Washington tinha como finalidade explorar o impacto a curto prazo da exposição a agrotóxicos na exacerbação da asma em crianças com asma em uma comunidade agrícola. Para o estudo obtiveram amostras repetidas de urina de um subconjunto de 16 crianças em idade escolar com asma. Através de exames de urina foram avaliados biomarcadores da exposição a agrotóxicos organofosforados (dialquilfosfatos) e exacerbação da asma (leucotrieno E4 - uLTE4). Deste modo, obteve-se 139 amostras de urina de 16 crianças durante o período. Verificou-se que 0 aumento dos níveis de uLTE4 está consistentemente associado ao aumento da exposição a organosfosforados. Concluiu-se que a exposição a curto prazo aos organofosforados está associada a um maior risco de morbidade da asma, como indicado pelo aumento dos níveis de uLTE4 nesta coorte de crianças com asma em uma comunidade agrícola (Benka-Coker, Loftus, Karr e Magzamen, 2019).

Um estudo transversal realizado no Distrito de Brebes, Indonésia teve como objetivo examinar a associação entre a exposição a agrotóxicos do grupo químico organofosforado e a ocorrência de disfunção tireoidiana em crianças. A metodologia empregada consistiu na coleta de amostras de urina e aplicação de questionários para os responsáveis pelas crianças. A análise de agrotóxicos nas amostras de urina contemplou um método cromatográfico de determinação multiresíduos. Observou-se que metabólitos de agrotóxicos organofosforados foram detectados em amostras de urina de $15(23 \%)$ de 66 crianças. Níveis de hormônio estimulador da tireoide (TSH) >4,5 IU/mL foram detectados em 24 (36\%) crianças. Os níveis de tiroxina livre (FT4) de todos os participantes eram normais. 0 nível de TSH em crianças com metabólitos positivos de agrotóxicos organofosfo- 


\section{EXPOSIÇÃO DE CRIANÇAS A AGROTÓXICOS: UMA REVISÃO INTEGRATIVA}

rados na urina $(7,74 \mu \mathrm{lU} / \mathrm{mL})$ foi significativamente $(p=0,005)$ maior do que naqueles que eram negativos $(4,34 \mu \mathrm{IU} / \mathrm{mL})$. Ainda, a prevalência de hipotireoidismo em crianças com metabólitos de agrotóxico organofosforado na urina $(67 \%)$ foi significativamente maior do que naqueles que eram negativos (27\%; RP 2,4; IC95\% 1,4 a 4,3). Neste sentido, os pesquisadores concluíram que uma exposição prolongada de crianças a agrotóxicos é considerada um fator de risco para a ocorrência da disfunção tireoidiana em crianças que vivem em áreas agrícolas (Suhartono et al., 2018).

0 estudo caso-controle de Ruiz-Guzmán, Gómez-Corales, Cruz-Esquivel, Marrugo- Negrete (2017) realizado nos municípios de Cereté, San Carlos, Montería, San Pelayo e cidade de Monteria, departamento de Córdoba, Colômbia tinha como objetivo avaliar a exposição a agrotóxicos e a frequência de danos citogenéticos em populações infantis em áreas agrícolas do departamento de Córdoba, Colômbia. Para este estudo coletou-se amostras de sangue para análise de micronúcleos e amostras de urina para determinação de Atrazina e seus metabólitos no organismo das crianças por meio de cromatografia gasosa após extração líquido-líquido. 0 estudo incluiu crianças entre 5 e 15 anos de idade de ambos os sexos com permanência mínima de três anos na área de estudo, sendo 50 crianças expostas e 13 não expostas. Verificou-se que concentrações mensuráveis de atrazina e/ou seus metabólitos foram registradas nas Zonas de Pelayito, Águas Negras e Cabuya, que apresentaram maior frequência de MN, botões nucleares e células apoptóticas do que no grupo controle. As maiores frequências de MN e células apoptóticas foram registradas em Pelayito, Águas Negras e Cabuya, sem diferenças significativas entre elas $(p>0,05)$, enquanto 0 Ceibita e 0 grupo controle registraram as taxas mais baixas sem diferença significativa. Não foram observadas correlações significativas $(p>0,05)$ entre os marcadores de danos citogenéticos (MN, botões nucleares e células apoptóticas) avaliadas e as concentrações de atrazina e seus metabólitos ( $p>0,05)$. Entretanto, concluiu-se que a frequência de danos citogenéticos no grupo que reside em áreas agrícolas (grupo exposto) é maior que no grupo controle.

Conforme o estudo realizado por Campos, da Silva, de Mello e Otero (2016), analisou- se a associação entre a exposição a agrotóxicos e a prevalência de transtornos mentais comuns e depressão autorreferida em um município do Rio Grande do Sul, Brasil, cuja principal atividade econômica é o cultivo de tabaco. Verificou-se que a exposição a agrotóxicos aos 15 anos de idade ou menos foi positivamente associada à depressão autorreferida. Esse resultado pode ser explicado pela maior vulnerabilidade de crianças e adolescentes à exposição a substâncias neurotóxicas, uma vez que o desenvolvimento do sistema nervoso central começa no período gestacional e se estende até a adolescência. Também, foi observado um aumento de $73 \%$ nas chances de exposição a agrotóxicos em idade igual ou inferior a 15 anos, entre os indivíduos que relataram depressão.

Um estudo realizado por Motta et al. (2016), tinha como objetivo avaliar o índice de contaminação de metais e agrotóxicos em mulheres grávidas e relacioná-lo a resultados perinatais, na área rural da região de Botucatu, São Paulo, Brasil. Analisou-se amostras de sangue de 40 mães e de seus recém-nascidos, por meio de resultados de Rudge. Os resultados obtidos indicam que não houve correlação estatisticamente positiva $(p>0,05)$ entre 0 índice de contaminação materna com os parâmetros clínicos dos recém-nascidos (RN) e o índice de contaminação do RN versus os parâmetros clínicos do RN (Motta et al., 2016).

Nascimento et al. (2017) avaliaram a saúde de crianças que vivem em uma região produtora de tabaco no Rio Grande do Sul, Brasil, através de diferentes biomarcadores de exposição e efeito, além de parâmetros hematológicos. Para tanto, amostras de sangue periférico e urina foram coletadas de 40 crianças de 6 a 12 anos, em dois períodos distintos: no início das aplicações de agrotóxicos e na colheita das folhas. Os resultados obtidos demonstram que crianças expostas ambientalmente a xenobióticos na área rural podem apresentar disfunção renal precoce, alterações hematológicas, 
além de danos aos lipídios e proteínas, associados à coexposição a diferentes xenobióticos envolvidos no cultivo do tabaco.

Um estudo de caso-controle de base populacional realizado em uma comunidade do norte da Itália teve como finalidade avaliar a possível relação entre exposição passiva a agrotóxicos agrícolas e risco de leucemia aguda na infância. Para tanto, avaliaram a exposição passiva a agrotóxicos de 111 casos de leucemia infantil e 444 controles correspondentes, determinando a densidade e 0 tipo de uso da terra agrícola dentro de um raio de $100 \mathrm{~m}$ em torno do lar das crianças. 0 foco foi determinado para quatro tipos comuns de culturas, arvenses, pomares, vinhedos e vegetais, caracterizados pelo uso de agrotóxicos específicos potencialmente envolvidos na leucemia induzida na infância. 0 risco de leucemia infantil não aumentou em relação a nenhum dos tipos de cultura, com exceção das culturas arvenses, caracterizadas pelo uso de 2,4-D, MCPA, glifosato, dicamba, triazina e cipermetrina. As poucas crianças $(n=11)$ que residem próximas a culturas arvenses apresentaram Odds Ratio (OR) para leucemia infantil de 2,04 (IC 95\% 0,50-8,35), e esse risco excessivo foi aumentado ainda mais em crianças com idade inferior a 5 anos (Malagoli et al., 2016).

Ismail et al. (2017) tiveram como objetivo comparar os parâmetros neurológicos resultados de duas coortes de adolescentes egípcios que trabalhavam como aplicadores de agrotóxicos. No ano de 2005 e 2009, duas coortes de adolescentes do sexo masculino que trabalhavam como aplicadores de agrotóxicos na colheita de algodão foram recrutados na província de Menoufia, no Egito. 0 mesmo cronograma de aplicação e agrotóxicos foram utilizados nos dois momentos. Os participantes de ambas as coortes completaram três testes neurocomportamentais, questionários de saúde e exposição e exames médicos e neurológicos de triagem. Além disso, foram coletadas amostras de sangue para medir a atividade da butiril colinesterase (BChE). Os aplicadores de agrotóxicos em ambas as coortes relataram mais sintomas e sinais neurológicos do que não aplicadores, particularmente entre os participantes da coorte de 2005 (OR variou de 1,18 a 15,3) exceto por um teste (Trail Making B), em que não houve diferenças significativas entre aplicadores ou não aplicadores de ambas as coortes nas medidas de resultados neurocomportamentais $(p>0,05)$. A coorte de 2005 mostrou maior inibição da atividade sérica de BChE do que a coorte de $2009(p<0,05)$. Além disso, os participantes com atividade reduzida de BChE mostraram mais sintomas e sinais do que outros sem redução do BChE $(p<0,05)$. Este estudo mostrou fortes evidências do impacto que a exposição a agrotóxicos causa na saúde de adolescentes.

0 estudo de Fiedler et al. (2015), teve como objetivo avaliar os efeitos neurocomportamentais de organofosforados (OP) e piretróides (PIR) na exposição de crianças entre 6 a 8 anos de idade. Cinquenta e quatro crianças tailandesas saudáveis foram selecionadas aleatoriamente entre 200 voluntários recrutados na região de arroz (25 crianças) e de aquicultura (24 crianças selecionadas para participar como grupo controle). Realizou-se a coleta de urina dessas crianças e a aplicação de um sistema de testes neurocomportamental computadorizado BARS (Sistema de Avaliação e Pesquisa Comportamental). Os participantes da fazenda de arroz apresentaram concentrações significativamente maiores de dialquilfosfatos (DAPs) (metabólitos comuns dos organofosforados (OPs)) e TCPy (um metabólito específico dos clorpirifós) do que as crianças da fazenda de aquicultura nas duas estações. Porém, os participantes das fazendas de arroz não tiveram desempenho significativamente pior em medidas neurocomportamentais do que os participantes das fazendas aquáticas durante as épocas de uso alto ou baixo de agrotóxicos. Não houve redução significativa no desempenho das crianças da fazenda de arroz nem na de aquicultura, mesmo que os metabólicos urinários das OPs tenham sido significativamente maiores na fazenda de arroz do que na de aquicultura. As comparações de grupo sugeriram uma melhora no desempenho do arroz em relação aos participantes da fazenda aquática durante a "baixa temporada de uso de pesticidas". Esse 


\section{EXPOSIÇÃO DE CRIANÇAS A AGROTÓXICOS: UMA REVISÃO INTEGRATIVA}

resultado pode ser interpretado para sugerir que, quando os pesticidas eram usados em maiores quantidades, o desempenho neurocomportamental dos participantes da fazenda de arroz era suprimido.

\section{CONCLUSÕES}

A literatura consultada traz importantes contribuições da produção científica sobre os impactos deletérios da exposição a agrotóxicos sobre a saúde de crianças que residem em áreas rurais. Esta revisão integrativa evidenciou que a exposição de crianças a agrotóxicos na zona rural está de fato associado a ocorrência de uma série de agravos a saúde infantil. Observou-se que no Brasil, líder mundial na utilização de agrotóxicos desde 2010, e que recentemente liberou uma série de outros produtos químicos proibidos em outros países, não apresenta estudos de coorte sobre o tema. Portanto, seria benéfico ver estudos adicionais no Brasil referente a exposição de crianças a agrotóxicos, de modo que os resultados evidenciem os perigos desses produtos a saúde de crianças para que então políticas públicas sejam desenvolvidas para dar mais atenção a saúde infantil.

\section{REFERÊNCIAS BIBLIOGRÁFICAS}

Barreto, S.; Herman, L.; Garibotti, V. (2012). Levantamento dos Agrotóxicos Usados no Estado do Rio Grande do Sul por Bacia Hidrográfica. Boletim Epidemiológico, 14(2), 3-6.

Benka-Coker, W. O., Loftus, C., Karr, C., Magzamen, S. (2019). Association of Organophosphate Pesticide Exposure and a Marker of Asthma Morbidity in an Agricultural Community. Journal of Agromedicine, 3, 1-9.

Campos, E., da Silva, V. S. P., de Mello, M. S. C., Otero, U. B. (2016). Exposure to pesticides and mental disorders in a rural population of Southern Brazil. NeuroToxicology, 56, 7-16.

Carneiro, F. F., Rigotto, R. M., Augusto, L. G. da S., Friedrich, K., Búrigo, A. C. (2015). Dossiê ABRASCO: um alerta sobre os impactos dos agrotóxicos na saúde. Rio de Janeiro, EPSJV; São Paulo, Expressão Popular, 624 p.

Cevs/Ses. Centro de Vigilância em Saúde da Secretaria da Saúde/Secretaria Estadual da Saúde. (2010). Relatório Final. Levantamento do uso e da criticidade dos agrotóxicos usados no Estado do Rio Grande do Sul. Porto Alegre, Talha-Mar Soluções Ambientais, 373.

Fiedler, N., Rohitrattana, J., Siriwong, W., Suttiwan, P., Strickland, P. O., Ryan, P. B., Rohlman, D. S., Panuwet, P., Barr, D. B., Robson, M. G. (2015). Neurobehavioral effects of exposure to organophosphates and pyrethroid pesticides among thai children. NeuroToxicology, 48, 90-99.

Ibama. Instituto Brasileiro do Meio Ambiente e dos Recursos Naturais Renováveis. (2018). Relatórios de Comercialização de Agrotóxicos. Recuperado de https://www.ibama.gov.br/agrotoxicos/relatorios-de-comercializacao-de-agrotoxicos\#

Ismail, A. A., Bonner, M. R., Hendy, O., Rasoul, G. A., Wang, K., Olson, J. R., Rohlman, D.

S. (2017). Comparison of neurological health outcomes between two adolescent cohorts exposed to pesticides in Egypt. PLOS ONE, 12, 1-13.

Kapka-Skrzypczak, L., Czajka, M., Sawicki, K., Matysiak-Kucharek, M., Gabelova, A., Saramkova, M., Bartyzel-Lechforowicz, H., Kruszewski, M. (2019). Assessment of DNA damage in Polish children environmentally exposed to pesticides. Mutation Research - Genetic Toxicology and Environmental Mutagenesis, 843, 52-56.

Karr, C (2012) Children's environmental health in agricultural settings. Journal Agromedicine, 17, 127-139.

Malagoli, C., Costanzini, S., Heck, J. E., Malavolti, M., de Girolamo, G., Oleari, P., Palazzi, G., Teggi, S., Vinceti, M. (2016). Passive exposure to agricultural pesticides and risk of childhood leuke- 
mia in an Italian community. International Journal of Hygiene and Environmental Health, 219, 742-748.

Miller, M. D., Marty, M. A., Landrigan, P. J. (2016). Children's environmental health. Pediatric Clinics of North America, 63, 149-165.

Motta, I. S., Volpato, G. T., Dmasceno, D. C., Sinzato, Y. K., Vesentini, G., Rudge, C. V. C.,

Calderon, I. M. P., Kempinas, W. de G., Odland, J. 0., Rudge, M. V. C. (2016). Contamination index. A novel parameter for metal and pesticide analyses in maternal blood and umbilical cord. Acta Cirúrgica Brasileira, 31(7), 490-497.

Nascimento, S. N., Göethel, G., Baierle, M., Barth, A., Brucker, N., Charão, M. F., Moro, A. M., Gauer, B., Sauer, E., Durgante, J., Arbo, M. D., Thiesen, F. V., Pierre, T. D. S., Gioda, A., Moresco, R., Garcia, S. C. (2017). Environmental exposure and effects on health of children from a tobaccoproducing region. Environmental Science and Pollution Research, 24, 2851-2865.

Perry, M. J (2003). Children's agricultural health: traumatic injuries and hazardous inorganic exposures. Summer, 19, 269-278.

Pignati, W. A., e Lima, F. A. N. de S., de Lara, S. S., Correa, M. L. M., Barbosa, J. R., Leão,

L. H. da C., Pignatti, M. G. (2017). Distribuição espacial do uso de agrotóxicos no Brasil: uma ferramenta para a vigilância em saúde. Ciência \& Saúde Coletiva, 22(10), 3281-3293.

Ruiz-Guzmán, J. A., Gómez-Corales, P., Cruz-Esquivel, Á., Marrugo-Negrete, J. L. (2017). Cytogenetic damage in peripheral blood lymphocytes of children exposed to pesticides in agricultural areas of the department of Cordoba, Colombia. Mutation Research Genetic Toxicology and Environmental Mutagenesis, 824, 25-31.

Sarcinelli, P. N. In: Peres, F., and Moreira, JC., (ORG). (2003). É veneno ou é remédio?: agrotóxicos, saúde e ambiente [online]. Rio de Janeiro, FIOCRUZ, 43-58.

Suhartono, S., Kartini, A., Subagio, H. W., Budiyono, Utari, A., Suratman, S., Sakundarno, M. (2018). Pesticide Exposure and Thyroid Function in Elementary School Children Living in an Agricultural Area, Brebes District, Indonesia. International Journal of Occupational and Environmental Medicine, 9(3), 137-144. 
